\title{
ESTUDIO HISTÓRICO-EPISTEMOLÓGICO DEL MODELO ATÓMICO DE RUTHERFORD
}

\author{
URIBE BELTRÁN M.V. \\ Maestría en Docencia de la Química, Universidad Pedagógica Nacional \\ Docente Universidad de La Sabana \\ CUÉLLAR FERNÁNDEZ L. \\ Maestría en Docencia de la Química, Universidad Pedagógica Nacional
}

\section{RESUMEN}

En este artículo se hace un análisis del modelo atómico de Rutherford desde el punto de vista histórico-epistemológico. La historia interna que se estudia empieza con el modelo atómico de Thomson, los problemas que presentó y la solución dada por el de Rutherford, y el modelo de Bohr, que resolvió algunas de las preguntas que se suscitaron a partir del modelo anterior, con la mirada desde la mecánica cuántica.

PALABRAS CLAVES Análisis Epistemológico, Historia Interna, Problema.

\section{ABSTRACT}

This article refers to the analysis of Rutherford's atomic model from an historicepistemologycal point of view. The internal history departs from the Thomson's atomic model, its problems and the solution gives by Rutherford's one, and the Bohr's model, which was worth to solve some of the remaining interrogants from the last one, from the quantum mechanic's point of view.

KEY WORDS Epistemologyc Analysis, Internal History, Problem.

\section{INTRODUCCIÓN}

El estudio del desarrollo histórico-epistemológico de las teorías o modelos científicos es hoy un campo de interés, no solo para los especialistas en este campo de conocimiento sino, también, para quienes se ocupan de los problemas de la didáctica de las ciencias experimentales, ya que esto les permite aproximarse a una comprensión de la dinámica de producción de conocimientos en este sector de la cultura universal, además de analizar los procesos de transposición didáctica (Chevallard,1998; Kang and Kilpatrick, 1992).

En el presente artículo se aborda el estudio retomando, principalmente, el concepto de historia de las ciencias formulado por I. Lakatos (1983), aún cuando se hace alusión a la concepción de T.S. Kuhn (1962), ambos conocidos como "epistemólogos-historicistas". En consecuencia, se toma distancia de las versiones historiográficas que aún siguen dominando en cierto sector del profesorado de ciencias.

Para adentrarse en el estudio que aquí se aboca es necesario centrarse en los artículos originales en los que los tres científicos, J.J. Thomson, E. Rutherford y N. Bohr, desarrollaron sus modelos atómicos (Thomson, 1904; Rutherford, 1911 y 1914; Bohr, 1913). La lectura de los mismos, además del dominio conceptual y metodológico necesario, precisa del establecimiento de una mirada epistemológica, tanto general como específica, por lo que se adoptó la lakatosiana (Lakatos, 1983). 
Como antecedentes, hay que decir que se contaba con la teoría atómica de John Dalton, propuesta en 1803, y que resultó fundamental para toda la investigación posterior en los campos de la Química y de la Física. En ese momento, la estructura general de esta teoría seguía siendo aceptada, pero con los trabajos de M. Faraday y de W. Crookes y el descubrimiento de la radiactividad se pusieron de manifiesto algunos aspectos del átomo que motivaron a un gran grupo de científicos a trabajar en este campo. Se concluyó que el átomo no podía ser una partícula indivisible, como lo propuso Dalton. Se hizo necesario reformular su teoría o modelo atómico y se inició una historia de reconceptualizaciones y elaboraciones de nuevos modelos, como se analizará a continuación.

Antes de entrar a desarrollar el propósito de este artículo, se considera indispensable discurrir en torno a lo que los autores del mismo van a considerar, tanto por modelo en las ciencias experimentales como por historia interna.

Dado que se hablará de modelo, los autores del presente artículo consideran importante referir a autores como T. S. Kuhn (1962), I. Lakatos (1983) y A. Badiou (1972) quienes han discurrido en torno a la categoría epistemológica de modelo, tanto en ciencias experimentales como en matemáticas. A. Badiou, cita a Gaston Bachelard, quien especifica que: "El modelo planetario de Bohr sólo hizo entrega de una imagen útil del átomo, en la época en que la microfísica acompañaba la borradura de sus órbitas y, finalmente, la renuncia de esa imagen, en beneficio de un modelo estadístico. Quien no sabía renunciar al modelo, renunciaba al saber: toda detención en un modelo conforma un obstáculo epistemológico ¿Hasta qué punto el modelo permanece al margen de la producción de conocimiento? Con todo, en ese lugar no es recusable, no presenta siquiera un problema".

No obstante, los autores del presente artículo, teniendo en cuenta la cita hecha sobre lo afirmado por Bachelard, se acogen a la concepción de N. R. Hanson (1977), para quien un modelo es una estructura conceptual que sugiere un marco de ideas para un conjunto de descripciones que de otra manera no serían sistematizables. Tal marco une las descripciones mediante lazos inferenciales.

En cuanto a la historia interna del desarrollo científico, retomando a I. Lakatos (1983), se dirige al análisis de las razones por las cuales una teoría o modelo científico fue propuesto, de la teoría o modelo que sustituyó, de la lógica interna de su estructura conceptual y metodológica; de los problemas que el anterior resolvió, de aquellos de los que no pudo dar cuenta y que crearon la necesidad del sustituto, como también de por qué el sustituto dio paso luego a otro.

\section{ANTECEDENTES}

Para entrar a caracterizar el modelo atómico de Thomson, precursor del modelo de Rutherford, es necesario mencionar las experiencias de Faraday con la electrólisis, alrededor de 1830, que hicieron pensar en la posibilidad de que el átomo no fuera indivisible y que la materia estuviera formada por partículas con carga eléctrica. Más adelante, a partir de 1870, Crookes determinó que cada gas daba en el espectroscopio un espectro propio, que debía ser consecuencia de cierta organización interior, que reforzaba la posibilidad de que el átomo se pudiera dividir. Una vez descubiertos los rayos catódicos y conocidas sus propiedades, como son que no dependen del tipo de gas encerrado en el tubo, que se desvían hacia el polo positivo cuando se someten a la acción de un campo eléctrico o magnético; que provoca la aparición de sombras y pone al rojo una barra de mica interpuesta en su camino y que la relación carga/masa no depende del gas, J.J. 
Thomson identificó esas partículas subatómicas; más adelante las llamó "electrones" (Thomson, 1906).

El descubrimiento de la radiactividad en 1896, por $\mathrm{H}$. Becquerel, y la observación de que la emisión espontánea de radiaciones aparecía tanto cuando estaba aislado el elemento como cuando estaba formando un compuesto, hizo pensar que provenía del interior del átomo y no de su parte externa, ya que no se acababan luego de una reacción química. Madame Curie había observado, antes de obtener el radio y el polonio, que el átomo tenía la radiactividad como propiedad intrínseca, y esto fue la clave para desarrollar la estructura atómica. En 1899 Ernest Rutherford publicó un artículo en el cual describía sus investigaciones sobre los nuevos rayos descubiertos por Becquerel y de los cuales observaba que no eran todos del mismo tipo; unos, que llamó rayos alfa, que eran muy rápidamente absorbidos por la materia y otros, a los que llamó beta, con un poder de penetración muy superior al de los alfa (Rutherford, 1899). Becquerel utilizó campos magnéticos, igual que lo había hecho Thomson para estudiar los rayos catódicos, y demostró que los rayos beta también se deflectaban con el campo magnético y que poseían la misma relación carga-masa de los electrones, llegando así a la conclusión de que los rayos beta estaban constituidos por electrones.

Aún no se sabía qué eran los rayos alfa, aunque parecía que no eran afectados por el campo magnético. Rutherford usó un campo magnético de mayor intensidad con el cual logró la deflexión y determinó que su carga era positiva. Explicó que su velocidad era muy grande y su masa muy superior a la de los electrones, motivos por los cuales era difícil desviarlos con campos magnéticos de baja intensidad. Con experimentos posteriores, Rutherford y varios colaboradores demostraron que las partículas alfa eran núcleos de átomos de helio con dos cargas eléctricas positivas (Rutherford and Royds, 1909). Más tarde, se descubrió que el radio emitía otro tipo de rayos (diferentes de los alfa y beta) que no eran desviados por fuerzas magnéticas, lo que llevó a suponer que no tenían carga, a los que llamaron gamma.

\section{ACERCA DE LA HISTORIA INTERNA}

J.J. Thomson (1904) lanzó su primera hipótesis sobre la estructura interna del átomo: El átomo estaba constituido por corpúsculos con carga eléctrica negativa uniformemente distribuidos en una esfera difusa de electricidad positiva, lo que explicaba su neutralidad eléctrica. La distribución de los corpúsculos dentro del átomo fue analizada matemáticamente, encontrando Thomson que lo más probable era que estuvieran dispuestos en anillos concéntricos dentro del átomo y calculó su número más probable en cada uno. El interés de Thomson, en apoyo de su modelo atómico, fue mostrar que las propiedades del átomo, por su estructura de anillos, eran similares en muchos aspectos a las que se le adjudicaban a los elementos químicos y que, en particular, dependían de su peso atómico de manera análoga a lo que expresaba la ley periódica.

\section{EXPERIMENTO DE GEIGER Y MARSDEN}

H. Geiger y E. Marsden (Geiger y Marsden, 1909) idearon un experimento en el que hicieron incidir partículas alfa sobre láminas metálicas lo suficientemente finas como para que fuese máxima la probabilidad de que las partículas solo fueran dispersadas por un único átomo durante el tiempo que tardaba en atravesar la lámina. Se esperaba, de acuerdo con el modelo de Thomson, que las partículas alfa, por ser de alta energía y de gran masa, solo sufrieran muy leves desviaciones al atravesar el átomo; pero se sorprendieron al observar que varias partículas golpearon la lámina y se volvieron atrás. 
Rutherford analizó el resultado del experimento anterior y llegó a la conclusión de que la dispersión hacia atrás debía ser consecuencia de una única colisión. Hizo cálculos y comprendió que era imposible que esto ocurriera, a menos que se pensara que los átomos tenían prácticamente toda su masa concentrada en un diminuto núcleo. En ese momento tuvo la idea de un átomo con un núcleo diminuto de gran masa y portador de carga positiva, rodeado por electrones con masa despreciable comparada con la del núcleo y en número suficiente para neutralizar la carga del núcleo (Rutherford, 1911). El átomo de Thomson no sería capaz de desviar el proyectil en un ángulo tan grande como los observados por Geiger y Marsden. El modelo atómico de Rutherford le permitió calcular el número de partículas alfa que debían ser desviadas en ángulos grandes por la lámina de oro, coincidiendo este número con los resultados experimentales de Marsden y Geiger.

Es interesante ver cómo un experimento diseñado para comprobar una teoría mostró inconsistencias en ella y generó su reformulación, permitiendo explicaciones que no se hubieran logrado desde la teoría anterior. Mantuvo de ella la neutralidad del átomo, y propuso una nueva distribución de las partículas subatómicas.

\section{LA PROPUESTA DE RUTHERFORD}

Con el bombardeo de partículas alfa emitidas por material radiactivo contra una lámina de oro de $10^{-5} \mathrm{~cm}$ de espesor (equivalente en su capacidad de detener a las partículas alfa a $1,6 \mathrm{~mm}$ de aire), ideado por Geiger y Marsden para confirmar el modelo atómico de Thomson, donde se vio que algunas de las partículas alfa, cerca de 1 en 20000 , se devolvían con un ángulo promedio de $90^{\circ}$ al pasar a través de la lámina de oro, pudo Rutherford deducir que la distribución y número de las partículas deflectadas en ángulos grandes no seguían la ley de probabilidad esperada. "Parece razonable suponer que la deflexión a través de un ángulo grande es debida a un encuentro atómico simple" (Rutherford, 1914). Esto le permitió proponer su modelo atómico en el cual se planteaba que el átomo contiene carga +Ne en su centro rodeada por una esfera eléctrica con una carga negativa igual uniformemente distribuida en una esfera de radio $\mathrm{R}$ (e es la unidad de carga fundamental, tomada como $4,54 \times 10^{-10}$ unidades E.S.). La fuerza eléctrica $\mathrm{X}$ y el potencial $\mathrm{V}$ a una distancia $\mathrm{r}$ desde el centro de un átomo hasta un punto por dentro del átomo está dada por:

$$
\begin{aligned}
& X=N e\left(\left(\left(1 / r^{2}\right)-\left(r / R^{3}\right)\right)\right. \\
& V=N e\left((1 / r)-(3 / 2 R)+\left(r^{2} / 2 R^{3}\right)\right)
\end{aligned}
$$

Una partícula $\alpha$ de masa $\mathrm{m}$, velocidad u y carga $\mathrm{E}$ que se dispara directamente hacia el centro de un átomo será desviada a una distancia b desde el centro, dada por:

$$
(1 / 2) m u^{2}=N \text { e } E\left((1 / b)-(3 / 2 R)+\left(b^{2} / 2 R^{3}\right)\right)
$$

Asumiendo que la carga central es $100 \mathrm{e}$, se puede calcular que el valor de b para una partícula $\alpha$ de velocidad $2,09 \times 10^{9} \mathrm{~cm} / \mathrm{s}$ es cerca de $3,4 \times 10^{-12} \mathrm{~cm}$. En este cálculo, b es muy pequeño comparado con $R$, que se considera del orden del radio del átomo, $10^{-8} \mathrm{~cm}$ aproximadamente, y la partícula $\alpha$ penetra muy cerca de la carga central, ignorando el campo debido a la distribución uniforme de carga negativa. La teoría no tiene en cuenta desviaciones debidas a los corpúsculos con carga negativa distribuidos uniformemente alrededor del núcleo. 
Si se supone que la velocidad de una partícula con carga positiva que pasa muy cerca al centro del átomo no cambia apreciablemente en su paso a través del átomo, su trayectoria varía inversamente al cuadrado de la distancia por la acción de la fuerza repulsiva, describiendo una hipérbola con el centro del átomo, como foco externo.

Sobre el ángulo de deflexión propuso que si un haz de partículas electrificadas cae normalmente sobre una lámina de materia de espesor $t$, excepto algunas que se deflectarán con un ángulo grande, la mayoría de ellas pasará a través de la lámina sin cambio apreciable de velocidad. Siendo $\mathrm{n}$ el número de átomos por unidad de volumen en el material, el número de colisiones de la partícula con el átomo de radio $R$ es $\pi R^{2} n t$. La probabilidad $m$ de penetrar un átomo a una distancia $p$ de su centro es dada por $m=\pi p^{2} n t$. La oportunidad dm de pasar dentro de un radio $p$ y $p+d p$ está dada por:

$$
\begin{aligned}
\mathrm{dm}= & 2 \pi p n t . d p=(\pi / 4) n \mathrm{nb}^{2} \cot \phi / 2 \operatorname{cosec}^{2} \phi / 2 d \phi \\
& \cot \phi / 2=2 p / b
\end{aligned}
$$

El valor de dm da la fracción del número total de partículas que son desviadas entre los ángulos $\phi$ y $\phi+\mathrm{d} \phi$, mientras que la fracción que es deflectada en ángulos mayores, $p$, está dada por

$$
p=(\pi / 4) n t b^{2} \cot ^{2} \phi / 2
$$

Si se llama $\mathbf{r}$ a la distancia entre la dirección de incidencia de los rayos $\alpha$ sobre el material bombardeado y la huella que deja sobre la pantalla de sulfuro de cinc, y $Q$ al número total de partículas bombardeadas contra el material, el número y de partículas por unidad de área deflectadas en un ángulo $\phi$ está dado por:

$$
y=Q d m / 2 \pi r^{2} \operatorname{sen} \phi d \phi=\left(n t b^{2} \cdot Q \cdot \operatorname{cosec}^{4} \phi / 2\right) / 16 r^{2}
$$

Siendo $b=2 \mathrm{NeE} / \mathrm{mu}^{2}$, el número de huellas por unidad de área de la pantalla de sulfuro de cinc a una distancia $r$ desde el punto de incidencia del rayo es proporcional a:

$\operatorname{Cosec}^{4} \phi / 2$ o $1 / \phi^{4}$ si $\phi$ es muy pequeña.

El espesor del material bombardeado, t, que debe ser muy pequeño.

La magnitud de las carga central $\mathrm{Ne}$.

$1 /\left(m u^{2}\right)^{2}$, o al inverso de la cuarta potencia de la velocidad si m es constante.

Obviamente se requiere que el espesor del material sea tan pequeño, que el rayo deba atravesar solamente un átomo en su recorrido.

Rutherford y su equipo repitieron varias veces el experimento de Geiger y Marsden utilizando láminas de otros metales y encontraron un acuerdo razonablemente bueno entre los resultados obtenidos y la teoría propuesta.

En resumen, de los datos de la dispersión de las partículas $\alpha$ previamente dados por Geiger se dedujo que el valor de la carga nuclear era igual a cerca de la mitad del peso atómico multiplicado por la carga electrónica. Los experimentos fueron empezados por Geiger y Marsden para probar la teoría (Geiger y Marsden, 1913). El método experimental general empleado por ellos consistió en permitir que un estrecho rayo de partículas 
cayera normalmente sobre una delgada capa de material y observar, por el método del centelleo, su dispersión a través de diferentes ángulos.

El conteo de muchos cientos de partículas fue un trabajo muy difícil y laborioso. Ellos encontraron que sus resultados eran muy acordes con la teoría. Cuando el espesor de la película era muy pequeña, la deflexión era directamente proporcional al espesor y variaba inversamente con la cuarta potencia de la velocidad de las partículas $\alpha$ incidentes. Se hizo un estudio especial sobre el número de partículas que se dispersaban en ángulos que variaban entre los 5 y los $150^{\circ}$. Aunque sobre este intervalo el número disminuía en la relación 200000 a 1, la relación entre el número y el ángulo coincidía con la teoría dentro del límite de error experimental. Geiger y Marsden encontraron que la deflexión en átomos de materiales diferentes era aproximadamente proporcional al cuadrado del peso atómico, mostrando que la carga sobre el núcleo estaba cercanamente proporcional al peso atómico. Por determinación del número de partículas $\alpha$ deflectadas desde películas delgadas de oro, ellos concluyeron que la carga del núcleo era igual a cerca de la mitad del peso atómico multiplicado por la carga electrónica. Teniendo en cuenta las dificultades de este experimento, el número actual puede no ser considerado correcto en más de un $20 \%$. Los resultados de Geiger y Marsden estuvieron así en completo acuerdo con las predicciones derivables del modelo de Rutherford.

Rutherford, luego de comparar los resultados experimentales desde su modelo, corroboró que el átomo consistía de un núcleo de pequeñísimas dimensiones con carga positiva en el que además se encuentra casi toda la masa del átomo, rodeado por una distribución de electrones, con carga negativa, que hacen que el átomo sea neutro, extendidos a distancias del núcleo comparables con los radios aceptados del átomo. Las deflexiones grandes de las partículas $\alpha$ y $\beta$ eran debidas al paso de ellas a través del campo de fuerza central; que estas deflexiones no podrían ocurrir con el átomo ideado por J.J. Thomson, a menos que el diámetro de la esfera que representaba cada átomo fuera excesivamente pequeña (Rutherford, 1911) .

\section{PROBLEMAS QUE PRESENTÓ EL MODELO DE RUTHERFORD}

El centro de interés de todo el grupo de Manchester era continuar en todas las direcciones posibles a partir de la postulación de la existencia del núcleo atómico. Recién llegado N. Bohr al laboratorio de Manchester, en 1912, se interesó por las implicaciones del núcleo atómico y por la distribución de electrones ligados a este en las propiedades físicas y químicas de diferentes sustancias. Veía claro que las desintegraciones radiactivas se explicaban estudiando la constitución del núcleo, mientras que las características ordinarias, físicas y químicas, de los elementos se debían a propiedades del sistema electrónico exterior. Incluso, ya se admitía que a causa de la gran masa del núcleo y a su pequeño tamaño comparado con el del átomo, la constitución del sistema electrónico dependía de la carga total del núcleo. Esto llevó a la idea de número atómico que, siendo un número entero, debía expresar la carga nuclear como múltiplo de una unidad elemental de electricidad.

En 1912 no habían empezado a trabajar ellos con las propiedades físicas y químicas de los elementos basadas en el modelo atómico de Rutherford. Sin embargo, era evidente que la estabilidad de los sistemas atómicos se contradecía con los fundamentos clásicos de la mecánica y la electrodinámica. Según la mecánica Newtoniana, ningún sistema de cargas puntuales admite un equilibrio estático estable, y de acuerdo con la electrodinámica de Maxwell, cualquier movimiento de los electrones alrededor del núcleo 
debería dar lugar a una disipación de energía en forma de radiación, acompañada de una continua contracción del sistema, por lo que los electrones terminarían en el núcleo.

La formulación del cuanto universal de acción de M. Planck (1900) mostró una limitación de las teorías físicas clásicas. A. Haas, en 1910, había intentado fijar las dimensiones y periodos de los movimientos de los electrones por medio de la ecuación de Planck entre energía y frecuencia de un oscilador armónico, sobre la base del modelo atómico de Thomson. En 1912, J. Nicholson utilizó momentos angulares cuantizados para explicar unas rayas que observaron en espectros de nebulosas estelares y $\mathrm{N}$. Bjerrum, en el mismo año, dio el primer paso para explicar los espectros moleculares mediante la ley general de combinación espectral. En este momento, ya Bohr estaba convencido de que la constitución electrónica del átomo de Rutherford estaba gobernada por el cuanto de acción. Esto lo explicaba porque la ecuación de Planck se podía aplicar a los electrones menos fuertemente unidos, los implicados en las propiedades químicas y ópticas de los elementos, y además se presentaban relaciones análogas para los electrones firmemente unidos al átomo. C.G. Barkla había detectado producción de radiación al bombardear con electrones átomos de diferentes elementos $\mathrm{y}$, más tarde en Cambridge, Whiddington tomó medidas de la energía necesaria para lograr esa radiación; sus resultados fueron acordes con lo esperado partiendo de una estimación de la máxima energía de enlace de un electrón que gira en una órbita de Planck alrededor de un núcleo con una carga dada por el número atómico. William Brown estudió los resultados de Whiddington y fue totalmente conciente de que estaban muy conectadas las radiación de Barkla y la ordenación de los elementos en la tabla de D.I. Mendeleiev. La explicación completa la dio, luego, H.G. Moseley con sus trabajos en Manchester (Bohr, 1913).

Bohr estuvo investigando teóricamente sobre el frenado de los rayos alfa y beta por los materiales, estudiado anteriormente por J.J. Thomson a partir de su propio modelo y por otros trabajos basados en el modelo de Rutherford, y se le ocurrió que la transferencia de energía desde las partículas a los electrones se podría tratar por analogía con la dispersión y absorción de radiaciones. Continuó trabajando en el papel del cuanto de acción en la constitución electrónica del átomo de Rutherford, explicando con él problemas de uniones moleculares y efectos radioactivos y magnéticos. Pero la cuestión de la estabilidad atómica exigía una base más firme para su explicación. Las leyes simples que gobiernan los espectros ópticos de los elementos le ofrecieron la clave para ir en busca de la explicación de la estabilidad atómica basándose en el átomo de Rutherford. H.A. Rowland y otros hicieron mediciones precisas de las longitudes de onda de las rayas espectrales; Balmer y Schuster realizaron contribuciones en este campo y, finalmente, Rydberg propuso una aclaración de las leyes espectrales generales muy ingeniosa. Según él, la frecuencia $(v)$ de cada raya del espectro de un elemento dado se podía representar con precisión en la forma $v=T^{\prime}-T^{\prime \prime}$, donde $T^{\prime} y T^{\prime \prime}$ son dos elementos de un conjunto de términos espectrales T característico del elemento.

Estos planteamientos desafiaban a la mecánica ordinaria. Lord Rayleigh había insistido en que cualquier relación general entre las frecuencias de los modos normales de vibración de un modelo mecánico debería ser cuadrática y no lineal respecto de estas frecuencias. De acuerdo con la electrodinámica clásica, las frecuencias de la radiación relacionada con el movimiento de los electrones deberían cambiar continuamente con la energía emitida y de acuerdo con esto, el átomo de Rutherford no podría presentar un espectro de rayas.

Bohr dedicó sus esfuerzos a intentar dar explicación sobre esto. Aceptó la idea de A. Einstein de los cuantos de luz o fotones de energía ( $h v$, donde $h$ es la constante de Planck) y supuso que cualquier emisión o absorción de radiación por el átomo es un 
proceso individual acompañado de una transferencia de energía $h\left(T^{\prime}-T^{\prime \prime}\right)$, interpretando el producto hT como la energía de ligadura de los electrones en cierto estado estable o estacionario del átomo. Así, en un proceso de emisión de energía se presenta la transición del electrón desde un nivel alto de energía a otro inferior, y en un proceso de absorción el átomo transita desde su estado fundamental hasta uno excitado. En el caso más sencillo, el átomo de hidrógeno, la fórmula $T_{n}=R / n^{2}$, donde $n$ es un número entero y $\mathrm{R}$ la constante de Rydberg, los términos quedan dados con precisión. Esto llevaba a una sucesión de valores decrecientes para la energía de ligadura del electrón en el átomo de hidrógeno; el electrón situado inicialmente a gran distancia del núcleo emite radiación y se va ubicando cada vez más cerca del núcleo con estados estacionarios de ligadura cada vez más firmes, caracterizados por valores cada vez más bajos de $\mathrm{n}$ hasta llegar al estado fundamental especificado por $n=1$.

\section{LA FORMULACIÓN DE BOHR}

La idea de Bohr fue tratar de establecer una íntima relación entre el modelo atómico de Rutherford y los datos espectrales. No lo pudo hacer, dado que, por una parte, la precisión sobre la carga y masa del electrón y del núcleo estaban apoyadas totalmente en fenómenos físicos basados en los principios de la mecánica y del electromagnetismo clásicos, y por otra, el postulado cuántico excluye la posibilidad de explicar la estabilidad del átomo con cualquier principio clásico. Enfocó su explicación utilizando modelos físicos clásicos para la clasificación de los estados estacionarios basados en las hipótesis originales de Planck respecto a los estados energéticos de un oscilador armónico. La comparación entre un oscilador de frecuencia dada y el movimiento de un electrón alrededor del núcleo con una frecuencia de revolución dependiente de la energía de ligadura ofrecía un buen punto de partida. Para cada uno de los estados estacionarios del átomo de hidrógeno, la acción integrada sobre un período orbital del electrón se identificaba con el producto $\mathrm{nh}$, lo que en el caso de órbitas circulares equivale a una cuantización del momento cinético en unidades $h / 2 \pi$. Esto implicaba fijar la constante de Rydberg en función de la carga, e, y la masa, m, del electrón y la constante de Planck de acuerdo con la fórmula $R=2 \pi^{2} \mathrm{~m} \mathrm{e}^{4} / \mathrm{h}^{3}$, lo que coincidía, con bastante precisión, con los valores empíricos de e, $\mathrm{m}$ y $\mathrm{h}$. Pero este fue solo un acercamiento, en razón de que surgían problemas teóricos por la incompatibilidad entre la mecánica clásica y la cuántica (Bohr, 1913).

En este momento el modelo atómico de Bohr, en el cual los electrones de un átomo se encuentran girando alrededor del núcleo en órbitas circulares, ocupando cada uno de ellos la órbita de menor energía posible, o sea, la más cercana al núcleo, había resuelto los problemas que se le observaban al modelo atómico de Rutherford; sin embargo, eran aún, también, muchos los problemas que surgían del análisis de este nuevo modelo.

\section{CONCLUSIONES}

Los modelos atómicos de Thomson, Rutherford y Bohr se desarrollaron por un método hipotético-deductivo. El progreso científico ocurrió mediante el cambio de programas de investigación que fueron resultando regresivos (Lakatos, 1983). En el artículo se ha hecho alusión al "grupo de Manchester", por lo que conformaron una comunidad científica ocupada de formular y resolver problemas específicos sobre la estructura del átomo (Kuhn, 1962).

Hay una serie de principios en los cuales se basaba el modelo atómico de Thomson y que continúan siendo básicos en el modelo de Rutherford. Algunos de estos son la aceptación 
del átomo como partícula mínima de un elemento, la neutralidad eléctrica del átomo y la presencia en él de partículas con carga negativa. Estos conceptos pueden asumirse como el núcleo firme inicial del programa de investigación fundado por Thomson, quien fue profesor de Rutherford y lo admitió en su grupo de trabajo, al cual se vinculó Bohr bajo la dirección del último.

El cinturón protector estuvo constituido, en primer lugar, por las hipótesis derivadas del modelo de Thomson, las cuales fueron corroboradas negativamente por el experimento de Geiger y Marsden, que a su vez dio paso a la propuesta del modelo de Rutherford, y a la modificación del núcleo firme inicial. El programa de investigación cambió, dado que el de Thomson se hizo regresivo. Fue necesario otro cinturón protector con nuevas hipótesis fundadas en la estructura matemática de la mecánica clásica.

El progreso científico dentro del grupo de Manchester va a continuar la dinámica anterior con la introducción de cambios en el núcleo firme, por cuanto Bohr introduce las formulaciones de la mecánica cuántica para postular su modelo semicuántico del átomo.

Es esta la lectura histórico-epistemológica que los responsables de este artículo han querido destacar y someter a consideración de la comunidad de especialistas.

\section{BIBLIOGRAFIA}

BADIOU, A., 1972, El Concepto de Modelo. Bases para una Epistemología Materialista de las Matemáticas, México, Siglo XXI Editores.

BOHR. N., 1913, On the Constitution of Atoms and Molecules, Philosophical Magazine, Series 6, vol. 26, p. 1-25.

CHEVALLARD, I., 1998, La Transposición Didáctica, Del Saber Sabio al saber Enseñado, Editorial Aique, Argentina.

GEIGER, H., MARSDEN, E., 1909, On a Diffuse Reflection of the $\alpha$-Particles, Proc. Roy. Soc., vol. 82, p. 495-500.

GEIGER, H., MARSDEN, E., 1913, The Laws of Deflexion of $\alpha$-Particles through Large Angles, Philosophical Magazine, Series 6, Volume 25, Number 148.

HANSON, N.R., 1977, Patrones de Descubrimiento, Observación y Explicación, Madrid, Alianza.

KANG, W., KILPATRICK, J., 1992, Didactic Transposition in Mathematic Textbooks, 12(1), 2-7.

KUHN, T.S., 1962, La Estructura de las Revoluciones Científicas, Fondo de Cultura Económica, traducido por A. Contín, México.

LAKATOS, I., 1983, La Metodología de los Programas de Investigación Científica, Alianza Editorial, Madrid.

RUTHERFORD, E., 1899, Uranium Radiation and the Electrical Conduction Produced by it, Philosophical Magazine, Series 5, xlvii, pp.109-163. 
RUTHERFORD, E., ROYDS, 1909, T., The Nature of the $\alpha$ Particle from Radioactive Substances, Philosophical Magazine, 17, pp. 281-286.

RUTHERFORD, E., 1911, The Scattering of $\alpha$ and $\beta$ Particles by Matter and the Structure of the Atom, Philosophical Magazine, Series 6, vol. 21, p. 669-688.

RUTHERFORD, E., 1914, The Structure of the Atom, Philosophical Magazine, Series 6, vol. 27, p. 488-498.

THOMSON, J.J., 1904, On the Structure of the Atom, Philosophical Magazine, Series 6, Volume 7, Number 39, p. 237-265.

THOMSON, J.J., 1906, On the Number of Corpuscles in an Atom, Philosophical Magazine, vol. 11, p. 769-781.

THOMSON, J.J., 1906, Nobel Lecture in Physics. 